\title{
Heavy Cycles in 2-Connected Weighted Graphs with Large Weighted Degree Sums
}

\author{
Bing Chen ${ }^{1}$, Shenggui Zhang ${ }^{1,2}$, and T.C. Edwin Cheng ${ }^{2}$ \\ ${ }^{1}$ Department of Applied Mathematics, Northwestern Polytechnical University, \\ Xi'an, Shaanxi 710072, P.R. China \\ 2 Department of Logistics, The Hong Kong Polytechnic University, \\ Hung Hom, Kowloon, Hong Kong
}

\begin{abstract}
In this paper, we prove that a 2-connected weighted graph $G$ contains either a Hamilton cycle or a cycle of weight at least $2 m / 3$ if it satisfies the following conditions: (1) $\sum_{i=1}^{3} d^{w}\left(v_{i}\right) \geq m$, where $v_{1}, v_{2}$ and $v_{3}$ are three pairwise nonadjacent vertices of $G$, and two of them are nonadjacent vertices of an induced claw or an induced modified claw; (2) In each induced claw and each induced modified claw of $G$, all edges have the same weight. This extends several previous results on the existence of heavy cycles in weighted graphs.
\end{abstract}

Keywords: Hamilton cycle, weighted graph, induced claw (modified claw.)

\section{Terminology and Notation}

We use Bondy and Murty $[3$ for terminology and notation not defined here and consider finite simple graphs only. Let $G$ be a graph. $G$ is called a weighted graph if each edge $e$ is assigned a nonnegative number $w(e)$, called the weight of $e$. For a subgraph $H$ of $G, V(H)$ and $E(H)$ denote the sets of vertices and edges of $H$, respectively. The weight of $H$ is defined by $w(H)=\sum_{e \in E(H)} w(e)$. For a vertex $v \in V, N_{H}(v)$ denotes the set, and $d_{H}(v)$ the number, of vertices in $H$ that are adjacent to $v$. We define the weighted degree of $v$ in $H$ by $d_{H}^{w}(v)=$ $\sum_{h \in N_{H}(v)} w(v h)$. When no confusion occurs, we will denote $N_{G}(v), d_{G}(v)$ and $d_{G}^{w}(v)$ by $N(v), d(v)$ and $d^{w}(v)$, respectively.

An unweighted graph can be regarded as a weighted graph in which each edge is assigned weight 1 . Thus, in an unweighted graph, $d^{w}(v)=d(v)$ for every vertex $v$, and the weight of a subgraph is simply the number of its edges.

The number of vertices in a maximum independent set of $G$ is denoted by $\alpha(G)$. If $G$ is noncomplete, then for a positive integer $k \leq \alpha(G)$ we denote by $\sigma_{k}(G)$ the minimum value of the degree sum of any $k$ pairwise nonadjacent vertices, and by $\sigma_{k}^{w}(G)$ the minimum value of the weighted degree sum of any $k$ pairwise nonadjacent vertices. If $k>\alpha(G)$, then both $\sigma_{k}(G)$ and $\sigma_{k}^{w}(G)$ are defined as $\infty$.

An $(x, y)$-path is a path connecting the two vertices $x$ and $y$. A $y$-path is a path which has $y$ as one of its end-vertices. The distance between two vertices

Y. Shi et al. (Eds.): ICCS 2007, Part III, LNCS 4489, pp. 338 346, 2007.

(C) Springer-Verlag Berlin Heidelberg 2007 
$x$ and $y$, denoted by $d(x, y)$, is the length of a shortest $(x, y)$-path. We call the graph $K_{1,3}$ a claw, and the graph $K_{1,3}+e$ ( $e$ is an edge between two nonadjacent vertices in the claw) a modified claw. For a graph $G$, if there exist three pairwise nonadjacent vertices in which two of them are nonadjacent in an induced claw or an induced modified claw, then by $\bar{\sigma}_{3}^{w}(G)$ we denote the minimum value of the weighted degree sum of such three pairwise nonadjacent vertices. Otherwise, $\bar{\sigma}_{3}^{w}(G)$ is defined as $\infty$. Clearly we have $\bar{\sigma}_{3}^{w}(G) \geq \sigma_{3}^{w}(G)$.

\section{Results}

In [8], Pósa gave a degree sum condition for the existence of long cycles in graphs. This result was generalized by the following two theorems along different lines.

Theorem A (Fan [5]). Let $G$ be a 2-connected graph such that $\max \{d(x), d(y) \mid$ $d(x, y)=2\} \geq c / 2$. Then $G$ contains either a Hamilton cycle or a cycle of length at least $c$.

Theorem B (Fournier \& Fraisse [6]). Let $G$ be a $k$-connected graph where $2 \leq k<\alpha(G)$, such that $\sigma_{k+1}(G) \geq m$. Then $G$ contains either a Hamilton cycle or a cycle of length at least $2 m /(k+1)$.

Bondy et al. 2] generalized the result of Pósa to weighted graph. In [10], it was showed that if one wants to give a generalization of Theorem A to weighted graphs, some extra conditions cannot be avoided. By adding two extra conditions, the authors gave a weighted generalization of Theorem A. Motivated by this result, by adding two same extra conditions, Zhang et al. [9] gave a weighted generalization of Theorem $\mathrm{B}$ in the case $k=2$.

Theorem 1 (Zhang et al. [9]). Let $G$ be a 2-connected weighted graph which satisfies: (1) $\sigma_{3}^{w}(G) \geq m ;(2) w(x z)=w(y z)$ for every vertex $z \in N(x) \cap$ $N(y)$ with $d(x, y)=2$; (3) In every triangle $T$ of $G$, either all edges of $T$ have different weights or all edges of $T$ have the same weight. Then $G$ contains either a Hamilton cycle or a cycle of weight at least $2 \mathrm{~m} / 3$.

Theorem A was further extended by the following result.

Theorem C (Bedrossian et al.[1]). Let $G$ be a 2-connected graph. If max $\{d(x), d(y)\} \geq c / 2$ for each pair of nonadjacent vertices $x$ and $y$, which are vertices of an induced claw or an induced modified claw of $G$, then $G$ contains either a Hamilton cycle or a cycle of length at least $c$.

By adding one extra condition, Fujisawa [7] gave a weighted generalization of Theorem C.

Theorem 2 (Fujisawa [7]). Let $G$ be a 2-connected weighted graph which satisfies: (1) For each induced claw and each induced modified claw of $G$, all its nonadjacent pair of vertices $x$ and $y$ satisfy $\max \left\{d^{w}(x), d^{w}(y)\right\} \geq s / 2$; (2) For each induced claw and each induced modified claw of $G$, all of its edges have the same weight. Then $G$ contains either a Hamilton cycle or a cycle of weight at least $s$. 
Clearly, Conditions (2) and (3) of Theorem 1 imply Condition (2) of Theorem 2. In a previous paper 4], the authors proved that Conditions (2) and (3) of Theorem 1 can be replaced by Condition (2) of Theorem 2. Here we will further show that Condition (1) of Theorem 1 can also be replaced by a weaker one.

Theorem 3. Let $G$ be a 2-connected weighted graph which satisfies the following conditions: (1) $\bar{\sigma}_{3}^{w}(G) \geq m$; (2) For each induced claw and each induced modified claw of $G$, all of its edges have the same weight. Then $G$ contains either a Hamilton cycle or a cycle of weight at least $2 \mathrm{~m} / 3$.

\section{Proof of Theorem 3}

We call a path $P$ a heaviest longest path of a graph $G$ if $P$ has the following properties

- $P$ is a longest path of $G$, and

- $w(P)$ is maximum among all longest paths in $G$.

Proposition 1. Let $G$ be a non-hamiltonian 2-connected weighted graph which satisfies conditions (1) and (2) of Theorem 3. Then $G$ contains a heaviest longest path $P=v_{1} v_{2} \cdots v_{p}$, such that $d^{w}\left(v_{1}\right)+d^{w}\left(v_{p}\right) \geq 2 m / 3$.

We prove this proposition in the next section. Theorem 3 can be proved by combining Proposition 1 and the following lemma. The proof of Lemma 1 is implicit in [2].

Lemma 1 (Bondy et al. [2]). Let $G$ be a non-hamiltonian 2-connected weighted graph and $P=v_{1} v_{2} \cdots v_{p}$ be a heaviest longest path in $G$. Then there is a cycle $C$ in $G$ with $w(C) \geq d^{w}\left(v_{1}\right)+d^{w}\left(v_{p}\right)$.

Proof of Theorem 3. Suppose that $G$ does not contain a Hamilton cycle. Then by Proposition 1 and Lemma 1, we can find a cycle of weight at least $2 \mathrm{~m} / 3$.

\section{Proof of Proposition 1}

In the proof of Proposition 1, we need the following two lemmas.

Lemma 2 (Fujisawa [7]). Let $G$ be a weighted graph satisfying Condition (2) of Theorem 3. If $x_{1} y x_{2}$ is an induced path with $w\left(x_{1} y\right) \neq w\left(x_{2} y\right)$ in $G$, then each vertex $x \in N(y) \backslash\left\{x_{1}, x_{2}\right\}$ is adjacent to both $x_{1}$ and $x_{2}$.

Lemma 3 (Fujisawa [7]). Let $G$ be a weighted graph satisfying Condition (2) of Theorem 3. Suppose $x_{1} y x_{2}$ is an induced path such that $w_{1}=w\left(x_{1} y\right)$ and $w_{2}=w\left(x_{2} y\right)$ with $w_{1} \neq w_{2}$, and $y z_{1} z_{2}$ is a path such that $\left\{z_{1}, z_{2}\right\} \cap\left\{x_{1}, x_{2}\right\}=\emptyset$ and $x_{2} z_{2} \notin E(G)$. Then the following $(i)$ and $(i i)$ hold:

(i) $\left\{z_{1} x_{1}, z_{1} x_{2}, z_{2} x_{1}\right\} \subseteq E(G)$, and $y z_{2} \notin E(G)$. Moreover, all edges in the subgraph induced by $\left\{x_{1}, y, x_{2}, z_{1}, z_{2}\right\}$, other than $x_{1} y$, have the same weight $w_{2}$. 
(ii) Let $Y$ be the component of $G-\left\{x_{2}, z_{1}, z_{2}\right\}$ with $y \in V(Y)$. For each vertex $v \in V(Y) \backslash\left\{x_{1}, y\right\}, v$ is adjacent to all of $x_{1}, x_{2}, y$ and $z_{2}$. Furthermore, $w\left(v x_{1}\right)=$ $w\left(v x_{2}\right)=w(v y)=w\left(v z_{2}\right)=w_{2}$.

Proof of Proposition 1. Choose a heaviest longest path $P=v_{1} v_{2} \cdots v_{p}$ in $G$ such that $d^{w}\left(v_{1}\right)+d^{w}\left(v_{p}\right)$ is as large as possible. It is clear that $N\left(v_{1}\right) \cup N\left(v_{p}\right) \subseteq$ $V(P)$. And it is not difficult to prove that there exists no cycle of length $p$.

Suppose $d^{w}\left(v_{1}\right)+d^{w}\left(v_{p}\right)<2 m / 3$. Without loss of generality, we may assume $d^{w}\left(v_{1}\right)<m / 3$.

Claim 1. Let $P_{1}$ and $P_{2}$ be two heaviest longest paths such that $P_{1}$ has $v^{\prime}$ and $v_{p}$ as its end-vertices, and $P_{2}$ has $v^{\prime \prime}$ and $v_{p}$ as its end-vertices. Then $v^{\prime}$ and $v^{\prime \prime}$ cannot be nonadjacent vertices of an induced claw or an induced modified claw.

Proof. Suppose $v^{\prime}$ and $v^{\prime \prime}$ are nonadjacent vertices of an induced claw or an induced modified claw. Since $P_{1}$ and $P_{2}$ are heaviest longest paths, $v^{\prime} v_{p} \notin E(G)$ and $v^{\prime \prime} v_{p} \notin E(G)$. By the choice of the path $P$ in $(\mathrm{b}), d^{w}\left(v^{\prime}\right) \leq d^{w}\left(v_{1}\right)$ and $d^{w}\left(v^{\prime \prime}\right)+d^{w}\left(v_{p}\right)<2 m / 3$. So we have $d^{w}\left(v^{\prime}\right)+d^{w}\left(v^{\prime \prime}\right)+d^{w}\left(v_{p}\right)<m$, contradicting Condition (1) of Theorem 3.

Since $G$ is 2-connected, $v_{1}$ is adjacent to at least one vertex on $P$ other than $v_{2}$. Choose $v_{k} \in N\left(v_{1}\right)$ such that $k$ is as large as possible. It is clear that $3 \leq k \leq$ $p-1$.

Case 1. $N\left(v_{1}\right)=\left\{v_{2}, \cdots, v_{k}\right\}$.

Since $P$ is longest and $N\left(v_{1}\right)=\left\{v_{2}, \cdots, v_{k}\right\}, N\left(v_{i}\right) \subseteq V(P)$ for every $i$ with $i=2, \cdots, k-1$; Since $G$ is a non-hamiltonian 2-connected graph, $k+2 \leq p$; Since $G-v_{k}$ is connected, there must be an edge $v_{j} v_{s} \in E(G)$ with $j<k<s$.

We assume that such an edge $v_{j} v_{s}$ was chosen so that: $(i) s$ is as large as possible; $($ ii $) j$ is as large as possible, subject to $(i)$. Clearly we have $s \leq p-1$.

Claim 2. $w\left(v_{1} v_{j+1}\right)=w\left(v_{j} v_{j+1}\right)$.

Proof. Suppose $j \leq k-2$. By the choices of $v_{k}, v_{j}$ and $v_{s}$, we have $v_{1} v_{s} \notin E(G)$ and $v_{j+1} v_{s} \notin E(G)$. So $\left\{v_{j}, v_{1}, v_{j+1}, v_{s}\right\}$ induces a modified claw, which implies that $w\left(v_{1} v_{j+1}\right)=w\left(v_{j} v_{j+1}\right)$.

Suppose $j=k-1$ and $s \geq k+2$. By the choices of $v_{k}$ and $v_{s}$, we have $v_{1} v_{s} \notin E(G), v_{1} v_{s+1} \notin E(G)$ and $v_{j} v_{s+1} \notin E(G)$. If $v_{k} v_{s+1} \in E(G)$, then $\left\{v_{k}, v_{1}, v_{k-1}, v_{s+1}\right\}$ induces a modified claw. So $w\left(v_{1} v_{k}\right)=w\left(v_{k-1} v_{k}\right)$. If $v_{k} v_{s} \notin$ $E(G)$, then $\left\{v_{k-1}, v_{1}, v_{k}, v_{s}\right\}$ induces a modified claw. So $w\left(v_{1} v_{k}\right)=w\left(v_{k-1} v_{k}\right)$. If $v_{k} v_{s+1} \notin E(G)$ and $v_{k} v_{s} \in E(G)$, then $\left\{v_{k}, v_{1}, v_{k+1}, v_{s}\right\}$ induces a claw or a modified claw. So $w\left(v_{1} v_{k}\right)=w\left(v_{k} v_{s}\right)$; On the other hand, $\left\{v_{s}, v_{k-1}, v_{k}, v_{s+1}\right\}$ induces a modified claw. So $w\left(v_{k} v_{s}\right)=w\left(v_{k-1} v_{k}\right)$; Then $w\left(v_{1} v_{k}\right)=w\left(v_{k-1} v_{k}\right)$.

Suppose $j=k-1$ and $s=k+1$. Since $P$ is longest, $N\left(v_{k}\right) \subset V(P)$; Since $G-v_{k+1}$ is connected, there exists an edge $v_{k} v_{t} \in E(G)$ with $t \geq k+2$. Choose $v_{k} v_{t}$ such that $t$ is as small as possible. So $\left\{v_{k}, v_{1}, v_{k-1}, v_{t}\right\}$ induces a modified claw. Then $w\left(v_{1} v_{k}\right)=w\left(v_{k-1} v_{k}\right)$. 
Claim 3. $s=k+1$.

Proof. Suppose $s \geq k+2$. Let $P^{\prime}=v_{s-1} v_{s-2} \cdots v_{j+1} v_{1} v_{2} \cdots v_{j} v_{s} v_{s+1} \cdots v_{p}$. If $v_{j} v_{s-1} \in E(G)$, then $\left\{v_{j}, v_{1}, v_{s-1}, v_{s}\right\}$ induces a modified claw, so $w\left(v_{s-1} v_{s}\right)=$ $w\left(v_{j} v_{s}\right)$. By Claim 2, now $P^{\prime}$ is a heaviest longest $v_{p}$-path different from $P$, contradicting Claim 1. If $v_{j} v_{s-1} \notin E(G)$, then $\left\{v_{s}, v_{j}, v_{s-1}, v_{s+1}\right\}$ induces a claw or a modified claw. Thus $w\left(v_{s-1} v_{s}\right)=w\left(v_{j} v_{s}\right)$. By Claim 2, both $v_{j} v_{j-1} \cdots v_{1}$ $v_{j+1} v_{j+2} \cdots v_{p}$ and $P^{\prime}$ are heaviest longest $v_{p}$-paths, contradicting Claim 1.

As in the proof of Claim 2, since $s=k+1$ and $G-v_{k+1}$ is connected, there exists an edge $v_{k} v_{t} \in E(G)$ with $k+2 \leq t \leq p-1$. Choose $v_{k} v_{t}$ such that $t$ is as small as possible.

Case 1.1. $t \geq k+3$.

By the choices of $v_{s}$ and $v_{t}, v_{j} v_{k+2} \notin E(G)$ and $v_{k} v_{k+2} \notin E(G)$. So $\left\{v_{k+1}, v_{j}\right.$, $\left.v_{k}, v_{k+2}\right\}$ induces a claw or a modified claw. Thus $w\left(v_{k} v_{k+1}\right)=w\left(v_{j} v_{k+1}\right)$.

Suppose $v_{k} v_{t+1} \notin E(G)$. By the choice of $v_{t}, v_{k} v_{t-1} \notin E(G)$. So $\left\{v_{t}, v_{k}, v_{t-1}\right.$, $\left.v_{t+1}\right\}$ induces a claw or a modified claw. Thus $w\left(v_{t-1} v_{t}\right)=w\left(v_{k} v_{t}\right)$. By Claim 2, both $v_{k} v_{k-1} \cdots v_{j+1} v_{1} \cdots v_{j} v_{k+1} \cdots v_{p}$ and $v_{t-1} v_{t-2} \cdots v_{k+1} v_{j} v_{j-1} \cdots v_{1} v_{j+1} \cdots$ $v_{k} v_{t} v_{t+1} \cdots v_{p}$ are heaviest longest $v_{p}$-paths, contradicting Claim 1. Suppose $v_{k} v_{t+1} \in E(G)$. By the choice of $v_{k}, v_{1} v_{t} \notin E(G)$ and $v_{1} v_{t+1} \notin E(G)$. So $\left\{v_{k}, v_{1}, v_{t}, v_{t+1}\right\}$ induces a modified claw. Thus $w\left(v_{k} v_{t+1}\right)=w\left(v_{t} v_{t+1}\right)$. By Claim 2, $v_{t} v_{t-1} \cdots v_{k+1} v_{j} v_{j-1} \cdots v_{1} v_{j+1} \cdots v_{k} v_{t+1} v_{t+2} \cdots v_{p}$ is a heaviest longest $v_{p^{-}}$ path different from $P$, contradicting Claim 1.

Case 1.2. $t=k+2$.

By the choice of $v_{k}$, now $\left\{v_{k}, v_{1}, v_{k+1}, v_{k+2}\right\}$ induces a modified claw. So we get $w\left(v_{k} v_{k+1}\right)=w\left(v_{k} v_{k+2}\right)=w\left(v_{k+1} v_{k+2}\right)=w\left(v_{1} v_{k}\right)$.

Since $G-v_{t}$ is connected, there exists an edge $v_{k+1} v_{t^{\prime}} \in E(G)$ with $t^{\prime} \geq k+3$. By the choice of $v_{s}, v_{j} v_{t} \notin E(G)$ and $v_{j} v_{t^{\prime}} \notin E(G)$. So $\left\{v_{s}, v_{j}, v_{t}, v_{t^{\prime}}\right\}$ induces a claw or a modified claw. In this case $s=k+1$ and $t=k+2$, thus we have $w\left(v_{j} v_{k+1}\right)=w\left(v_{k+1} v_{k+2}\right)$. By Claim 2 , now $v_{k+1} v_{j} v_{j-1} \cdots v_{1} v_{j+1} v_{j+2} \cdots v_{k} v_{k+2}$ $\cdots v_{p}$ is a heaviest longest $v_{p}$-path different from $P$. At the same time, $v_{1}$ and $v_{k+1}$ are nonadjacent vertices in the modified claw induced by $\left\{v_{k}, v_{1}, v_{k+1}, v_{k+2}\right\}$, contradicting Claim 1.

This completes the proof of Case 1 .

Case 2. $N\left(v_{1}\right) \neq\left\{v_{2}, \cdots, v_{k}\right\}$.

Choose $v_{r} \notin N\left(v_{1}\right)$ with $2<r<k$ such that $r$ is as large as possible. Then $v_{1} v_{i} \in E(G)$ for every $i$ with $r<i \leq k$. Let $j$ be the smallest index such that $j>r$ and $v_{j} \notin N\left(v_{1}\right) \cap N\left(v_{r}\right)$. Since $v_{r+1} \in N\left(v_{1}\right) \cap N\left(v_{r}\right)$, we have $j \geq r+2$. On the other hand, it is obvious that $j \leq k+1$. By the choice of $v_{r}$ and $v_{j}$, now $v_{1}$ and $v_{r}$ are nonadjacent vertices of a claw or a modified claw induced by $\left\{v_{j-1}, v_{1}, v_{r}, v_{j}\right\}$. By Claim 1 and the choice of $P$, we have the following claim.

Claim 4. There exists no heaviest longest $v_{p}$-path with $v_{r}$ as it's another endvertex.

Claim 5. $w\left(v_{1} v_{r+1}\right) \neq w\left(v_{r} v_{r+1}\right)$. 
Proof. If $w\left(v_{1} v_{r+1}\right)=w\left(v_{r} v_{r+1}\right)$, then $v_{r} v_{r-1} \cdots v_{1} v_{r+1} v_{r+2} \cdots v_{p}$ is a heaviest longest $v_{p}$-path different from $P$, contradicting Claim 4.

Claim 6. $v_{r+1} v_{j} \notin E(G)$.

Proof. By the choice of $v_{r}, v_{1} v_{r} \notin E(G)$. So, if $v_{r+1} v_{j} \in E(G)$, then by the choice of $v_{j}$, we know that $\left\{v_{r+1}, v_{1}, v_{r}, v_{j}\right\}$ induces a claw or a modified claw. Thus $w\left(v_{1} v_{r+1}\right)=w\left(v_{r} v_{r+1}\right)$, contradicting Claim 5 .

By Claim 6, we have $r+3 \leq j \leq k+1$. Let $n$ be the largest index such that $r+1 \leq n \leq j-2$ and $v_{n} v_{j} \notin E(G)$. Then $v_{i} v_{j} \in E(G)$ for every $i$ with $n+1 \leq i \leq j-1$.

Claim 7. If $r+3 \leq j \leq k$, then $w\left(v_{1} v_{n+1}\right)=w\left(v_{n} v_{n+1}\right)$.

Proof. By the choice of $v_{n}, v_{n+1} v_{j} \in E(G)$. So $\left\{v_{n+1}, v_{1}, v_{r}, v_{j}\right\}$ induces a modified claw. Thus $w\left(v_{1} v_{n+1}\right)=w\left(v_{n+1} v_{j}\right)$. Since $v_{r} v_{j} \notin E(G)$ and $v_{n} v_{j} \notin E(G)$, $\left\{v_{r}, v_{n}, v_{n+1}, v_{j}\right\}$ induces a modified claw. Thus $w\left(v_{n} v_{n+1}\right)=w\left(v_{n+1} v_{j}\right)$. So $w\left(v_{1} v_{n+1}\right)=w\left(v_{n} v_{n+1}\right)$.

Case 2.1. $r+3 \leq j \leq k-1$.

Suppose $v_{r+1} v_{j+1} \notin E(G)$. By Claim $6, v_{r+1} v_{j} \notin E(G)$, so $\left\{v_{1}, v_{j}, v_{j+1}, v_{r+1}\right\}$ induces a modified claw. Thus $w\left(v_{1} v_{j+1}\right)=w\left(v_{j} v_{j+1}\right)$. Suppose $v_{r+1} v_{j+1} \in$ $E(G)$. By Claim 5 and the choice of $v_{j}$, applying Lemma $3(i)$ to $\left\{v_{1}, v_{r+1}, v_{r}\right.$, $\left.v_{j+1}, v_{j}\right\}$, we get $w\left(v_{1} v_{j+1}\right)=w\left(v_{j} v_{j+1}\right)$.

By Claim 7 , both $v_{n} v_{n-1} \cdots v_{1} v_{n+1} v_{n+2} \cdots v_{p}$ and $v_{j} v_{j-1} \cdots v_{1} v_{j+1} v_{j+2} \cdots v_{p}$ are heaviest longest $v_{p}$-paths. Furthermore, $v_{n}$ and $v_{j}$ are nonadjacent vertices in the modified claw induced by $\left\{v_{r}, v_{n}, v_{n+1}, v_{j}\right\}$, contradicting Claim 1 .

Case $2.2 j=k$.

Since $\left\{v_{j-1}, v_{1}, v_{r}, v_{j}\right\}$ induces a claw or a modified claw and $j=k$, we have

Claim 8. $w\left(v_{1} v_{k-1}\right)=w\left(v_{1} v_{k}\right)=w\left(v_{k-1} v_{k}\right)$.

Claim 9. $v_{r+2} v_{k} \notin E(G)$.

Proof. Suppose $v_{r+2} v_{k} \in E(G)$. Applying Lemma $3(i i)$ to $\left\{v_{1}, v_{r+1}, v_{r}, v_{r+2}, v_{k}\right\}$ and the vertex $v_{2}$ (which is adjacent to $v_{1}$ ), we get $v_{2} v_{r+1} \in E(G)$ and $w\left(v_{1} v_{2}\right)=$ $w\left(v_{2} v_{r+1}\right)=w\left(v_{1} v_{k}\right)=w\left(v_{r} v_{r+1}\right)$. By Claim 8, $v_{r} v_{r-1} \cdots v_{2} v_{r+1} v_{r+2} \cdots v_{k-1} v_{1}$ $v_{k} v_{k+1} \cdots v_{p}$ is a heaviest longest $v_{p}$-path, contradicting Claim 4.

Now, we have $v_{r+1} v_{k-1} \notin E(G)$. Since otherwise, applying Lemma 3 (ii) to $\left\{v_{1}, v_{r+1}, v_{r}, v_{k-1}, v_{k}\right\}$ and the vertex $v_{r+2}$ (which is adjacent to $v_{r+1}$ ), we get $v_{r+2} v_{k} \in E(G)$, contradicting Claim 9. By Claim 6, $v_{k-1}$ and $v_{r+1}$ are nonadjacent vertices in the modified claw induced by $\left\{v_{1}, v_{r+1}, v_{k-1}, v_{k}\right\}$.

By Claims 6 and 9, now $\left\{v_{1}, v_{r+1}, v_{r+2}, v_{k}\right\}$ induces a modified claw. Then $w\left(v_{1} v_{r+2}\right)=w\left(v_{r+1} v_{r+2}\right)$. By Claim 8, both $v_{k-1} v_{k-2} \cdots v_{1} v_{k} v_{k+1} \cdots v_{p}$ and $v_{r+1} v_{r} \cdots v_{1} v_{r+2} \cdots v_{p}$ are heaviest longest $v_{p}$-paths, contradicting Claim 1 . 
Case $2.3 j=k+1$.

Since $\left\{v_{j-1}, v_{1}, v_{r}, v_{j}\right\}$ induces a claw or a modified claw, we have

Claim 10. $w\left(v_{1} v_{k}\right)=w\left(v_{k} v_{k+1}\right)=w\left(v_{r} v_{k}\right)$.

Claim 11. $v_{r+1} v_{k} \notin E(G)$.

Proof. Suppose $v_{r+1} v_{k} \in E(G)$. Applying Lemma $3(i i)$ to $\left\{v_{1}, v_{r+1}, v_{r}, v_{k}, v_{k+1}\right\}$ and the vertex $v_{r-1}$ (which is adjacent to $v_{r}$ ), we get $v_{r-1} v_{k+1} \in E(G)$ and $w\left(v_{r-1} v_{r}\right)=w\left(v_{r-1} v_{k+1}\right)$. By Claim 10, we get a heaviest longest $v_{p}$-path $v_{r} v_{r+1} \cdots v_{k} v_{1} v_{2} \cdots v_{r-1} v_{k+1} v_{k+2} \cdots v_{p}$, contradicting Claim 4.

Claim 12. $v_{i} v_{k+1} \notin E(G)$ for every $v_{i} \in N\left(v_{1}\right) \cap N\left(v_{r}\right) \backslash\left\{v_{k}\right\}$.

Proof. Suppose there exists a vertex $v_{i} \in N\left(v_{1}\right) \cap N\left(v_{r}\right) \backslash\left\{v_{k}\right\}$ such that $v_{i} v_{k+1} \in$ $E(G)$. If $w\left(v_{1} v_{r+1}\right) \neq w\left(v_{1} v_{k}\right)$, then applying Lemma $3(i)$ to $\left\{v_{r+1}, v_{1}, v_{k}, v_{i}\right.$, $\left.v_{k+1}\right\}$, we get $w\left(v_{1} v_{r+1}\right)=w\left(v_{k} v_{k+1}\right)$. So $w\left(v_{1} v_{k}\right) \neq w\left(v_{k} v_{k+1}\right)$, contradicting Claim 10. So we get $w\left(v_{1} v_{r+1}\right)=w\left(v_{1} v_{k}\right)$. Similarly, we can prove that $w\left(v_{r} v_{r+1}\right)=w\left(v_{r} v_{k}\right)$. By Claim 10, we get $w\left(v_{1} v_{r+1}\right)=w\left(v_{r} v_{r+1}\right)$, contradicting Claim 5.

Claim 13. $w\left(v_{1} v_{k-1}\right)=w\left(v_{k-1} v_{k}\right)$.

Proof. By the choice of $v_{k}$ and Claim 12, we get $v_{1} v_{k+1} \notin E(G)$ and $v_{k-1} v_{k+1} \notin$ $E(G)$. So $\left\{v_{k}, v_{1}, v_{k-1}, v_{k+1}\right\}$ induces a modified claw and $w\left(v_{1} v_{k-1}\right)=w\left(v_{k-1} v_{k}\right)$.

Case 2.3.1. $w\left(v_{r} v_{r+1}\right) \neq w\left(v_{r} v_{k}\right)$.

By Claim 11, $v_{r+1} v_{k} \notin E(G)$. Furthermore, $v_{r} v_{k+1} \notin E(G)$, since otherwise it follows from Lemma 2 that $v_{r+1} v_{k+1} \in E(G)$, which contradicts Claim 6 .

Applying Lemma 2 to the induced path $v_{r+1} v_{r} v_{k}$ and the vertex $v_{r-1} \in$ $N\left(v_{r}\right) \backslash\left\{v_{r+1}, v_{k}\right\}$, we get $v_{r-1} v_{r+1} \in E(G)$ and $v_{r-1} v_{k} \in E(G)$. By Claim $5, w\left(v_{1} v_{r+1}\right) \neq w\left(v_{r} v_{r+1}\right)$. Applying Lemma 2 to the induced path $v_{1} v_{r+1} v_{r}$ and the vertex $v_{r-1} \in N\left(v_{r+1}\right) \backslash\left\{v_{1}, v_{r}\right\}$, we get $v_{1} v_{r-1} \in E(G)$. This implies that $v_{r-1} \in N\left(v_{1}\right) \cap N\left(v_{r}\right)$. By Claim 12, we have $v_{r-1} v_{k+1} \notin E(G)$. Then $\left\{v_{k}, v_{r-1}, v_{r}, v_{k+1}\right\}$ induces a modified claw, so $w\left(v_{r-1} v_{r}\right)=w\left(v_{r-1} v_{k}\right)$. Therefore, by Claim 13, $v_{r} v_{r+1} \cdots v_{k-1} v_{1} v_{2} \cdots v_{r-1} v_{k} v_{k+1} \cdots v_{p}$ is a heaviest longest $v_{p}$-path, contradicting Claim 4.

Case 2.3.2. $w\left(v_{r} v_{r+1}\right)=w\left(v_{r} v_{k}\right)$.

By Claim 5 and Claim 10, we can get that $w\left(v_{1} v_{r+1}\right) \neq w\left(v_{1} v_{k}\right)$.

Claim 14. $v_{2} v_{k}, v_{2} v_{r+1}$ and $v_{2} v_{r} \in E(G)$.

Proof. By Claim 11, $v_{r+1} v_{k} \notin E(G)$. Applying Lemma 2 to the induced path $v_{r+1} v_{1} v_{k}$ and the vertex $v_{2} \in N\left(v_{1}\right) \backslash\left\{v_{r+1}, v_{k}\right\}$, we get $v_{2} v_{k} \in E(G)$ and $v_{2} v_{r+1} \in E(G)$. By Claim 5, w( $\left.v_{1} v_{r+1}\right) \neq w\left(v_{r} v_{r+1}\right)$. Applying Lemma 2 to the induced path $v_{1} v_{r+1} v_{r}$ and the vertex $v_{2} \in N\left(v_{r+1}\right) \backslash\left\{v_{1}, v_{r}\right\}$, we get $v_{2} v_{r} \in$ $E(G)$. 
Claim 15. $v_{r} v_{k+1} \notin E(G)$.

Proof. Suppose $v_{r} v_{k+1} \in E(G)$. By Claim 14, we get $v_{2} \in N\left(v_{1}\right) \cap N\left(v_{r}\right)$, $v_{2} v_{k}$ and $v_{2} v_{r+1} \in E(G)$. By Claims 6 and 12, we get $v_{r+1} v_{k+1} \notin E(G)$ and $v_{2} v_{k+1} \notin E(G)$. Thus both $\left\{v_{r}, v_{2}, v_{r+1}, v_{k+1}\right\}$ and $\left\{v_{k}, v_{1}, v_{2}, v_{k+1}\right\}$ induce modified claws. Then we have $w\left(v_{2} v_{r+1}\right)=w\left(v_{r} v_{r+1}\right)=w\left(v_{1} v_{2}\right)=w\left(v_{1} v_{k}\right)$. Ву Claim 13, we get $w\left(v_{1} v_{k-1}\right)=w\left(v_{k-1} v_{k}\right)$. So, $v_{r} v_{r-1} \cdots v_{2} v_{r+1} v_{r+2} \cdots v_{k-1} v_{1} v_{k}$ $v_{k+1} \cdots v_{p}$ is a heaviest longest $v_{p}$-path, contradicting Claim 4.

Suppose $v_{1} v_{r-1} \in E(G)$. Then by applying Lemma 2 to the induced path $v_{k} v_{1} v_{r+1}$ and $v_{r-1} \in N\left(v_{1}\right) \backslash\left\{v_{k}, v_{r+1}\right\}$, we get $v_{r-1} v_{k} \in E(G)$ and $v_{r-1} v_{r+1} \in$ $E(G)$. By Claims 12 and 15, we get $v_{r-1} v_{k+1} \notin E(G)$ and $v_{r} v_{k+1} \notin E(G)$. Then $\left\{v_{k}, v_{r-1}, v_{r}, v_{k+1}\right\}$ induces a modified claw. Thus $w\left(v_{r-1} v_{r}\right)=w\left(v_{r-1} v_{k}\right)$. Therefore, by Claim 13, we have $v_{r} v_{r+1} \cdots v_{k-1} v_{1} v_{2} \cdots v_{r-1} v_{k} v_{k+1} \cdots v_{p}$ is a heaviest longest $v_{p}$-path, contradicting Claim 4.

Suppose $v_{1} v_{r-1} \notin E(G)$. Then by Claim 5 and Lemma 2, we have $v_{r-1} v_{r+1} \notin$ $E(G)$. Furthermore, $v_{2} v_{r-1} \notin E(G)$, since otherwise, applying Lemma 3 (ii) to $\left\{v_{r}, v_{r+1}, v_{1}, v_{2}, v_{r-1}\right\}$ and the vertex $v_{k}$ (which is adjacent to $v_{r}$ ), we get $w\left(v_{1} v_{r+1}\right)=w\left(v_{1} v_{k}\right)$, a contradiction. By Claims 12 and $14, v_{2} v_{k+1} \notin E(G)$. Then, both $\left\{v_{r}, v_{r+1}, v_{2}, v_{r-1}\right\}$ and $\left\{v_{k}, v_{1}, v_{2}, v_{k+1}\right\}$ induce modified claws. Thus $w\left(v_{2} v_{r+1}\right)=w\left(v_{r} v_{r+1}\right)$ and $w\left(v_{1} v_{2}\right)=w\left(v_{1} v_{k}\right)$. By Claim 13, we know that $v_{r} v_{r-1} \cdots v_{2} v_{r+1} v_{r+2} \cdots v_{k-1} v_{1} v_{k} v_{k+1} \cdots v_{p}$ is a heaviest longest $v_{p}$-path, contradicting Claim 4.

The proof of the theorem is complete.

\section{Acknowledgements}

This work was supported by NSFC (No. 60642002). The second and the third authors were supported by The Hong Kong Polytechnic University under grant number G-YX42.

\section{References}

1. Bedrossian, P., Chen, G., Schelp, R.H.: A generalization of Fan's condition for Hamiltonicity, pancyclicity, and Hamiltonian connectedness, Discrete Math. 115 (1993) 39-50

2. Bondy, J.A., Broersma, H.J., van den Heuvel, J., Veldman, H.J.: Heavy cycles in weighted graphs, Discuss. Math. Graph Theory 22 (2002) 7-15

3. Bondy, J.A., Murty, U.S.R.: Graph Theory with Applications, New York: Macmillan London and Elsevier, 1976

4. Chen, B., Zhang, S.: A new $\sigma_{3}$-type condition for heavy cycles in weighted graphs, Accepted by Ars Combinatoria.

5. Fan, G.: New sufficient conditions for cycles in graphs, J. Combin. Theory Ser. B 37 (1984) 221-227

6. Fournier, I., Fraisse, P.: On a conjecture of Bondy, J. Combin. Theory Ser. B 39 (1985), 17-26. 
7. Fujisawa, J.: Claw conditions for heavy cycles in weighted graphs, Graphs \& Combin. 21 (2) (2005) 217-229

8. Pósa, L.: On the circuits of finite graphs, Magyar Tud. Math. Kutató Int. Közl. 8 (1963) 355-361

9. Zhang, S., Broersma, H.J., Li, X.: A $\sigma_{3}$ type condition for heavy cycles in weighted graphs, Discuss. Math. Graph Theory 21 (2001) 159-166

10. Zhang, S., Broersma, H.J., Li, X., Wang, L.: A Fan type condition for heavy cycles in weighted graphs, Graphs \& Combin. 18 (2002) 193-200 